\title{
Surgical cytoreduction of the primary tumor reduces metastatic progression in a mouse model of prostate cancer
}

\author{
FEDERICO F. CIFUENTES ${ }^{1,2}$, RODRIGO H. VALENZUELA ${ }^{1}$, \\ HÉCTOR R. CONTRERAS ${ }^{1}$ and ENRIQUE A. CASTELLÓN ${ }^{1}$
}

\begin{abstract}
${ }^{1}$ Laboratory of Molecular and Cellular Andrology, Physiology and Biophysics Program, Institute of Biomedical Sciences, Faculty of Medicine, University of Chile, Santiago 8380453; ${ }^{2}$ Department of Animal Pathology, Faculty of Veterinary and Animal Sciences, University of Chile, Santiago 8820808, Chile
\end{abstract}

Received June 18, 2015; Accepted July 30, 2015

DOI: 10.3892/or.2015.4319

\begin{abstract}
Metastatic prostate cancer ( $\mathrm{mPCa}$ ) is one of the most prevalent cancers in men worldwide. The main cause of death in these patients is androgen-resistant metastatic disease. Surgery of the primary tumor has been avoided in these patients as there is no strong evidence that supports a beneficial effect. From the biological point of view, it appears rational to hypothesize that the primary tumor may contribute to the establishment and growth of metastases. Considering this, we propose that cytoreductive surgery (CS) in advanced metastatic stage slows the progression of metastatic disease. To test this, we used a mouse model of resectable orthotopic prostate cancer ( $\mathrm{PCa}$ ) and performed CS. After surgery, metastases were smaller and less numerous in the treated mice; an effect that was observable until the end of the experiment. These results suggest that CS alone delays the progression of metastatic disease and that although this effect may be temporary, it may translate to prolonged survival, especially when used with adjuvant therapy.
\end{abstract}

\section{Introduction}

Prostate cancer ( $\mathrm{PCa}$ ) is the second leading cause of male tumor-related deaths in developed countries (1). The main treatment used in patients with metastatic prostate cancer $(\mathrm{mPCa})$ is androgen deprivation therapy which has demonstrated an improved overall survival after prostatespecific antigen detection (2). However, these patients often enter in an androgen-independent stage associated with high mortality (3). There are several treatment options for these patients, but none includes surgical management of the

Correspondence to: Dr Enrique A. Castellón, Laboratory of Molecular and Cellular Andrology, Physiology and Biophysics Program, Institute of Biomedical Sciences, Faculty of Medicine, University of Chile, Independencia 1027, Santiago 8380453, Chile E-mail: ecastell@med.uchile.cl

Key words: prostate cancer, metastasis, cytoreductive surgery primary tumor outside of a clinical trial setting $(3,4)$. Surgeons are becoming more prone to surgical treatment in high risk and locally advanced disease as a part of a multimodality approach to the treatment of $\mathrm{PCa}$, including the possibility to treat the primary tumor in M1 patients (4). There has arisen a question of whether removing the primary tumor in patients with metastatic disease is relevant, and during the last decade it has been addressed in several reports that analyze information available in retrospective studies $(5,6)$. Currently, cytoreductive surgery (CS) is used rarely in $\mathrm{mPCa}$ patients but only to relieve the patient from local side-effects derived from tumor growth (7) and not in the context of PCa treatment (3). The effect of a reduction in tumor mass in a patient presenting with metastasis may be used as an additional therapeutic measure to prolong patient survival, but there are no current pre-clinical models that confirm or refuse this hypothesis (4). From the biological point of view, the primary tumor has an important role in initiating and maintaining the metastatic process. The tumor has the propensity for constantly delivering cells into circulation $(8,9)$ while it also can prepare the distant pre-metastatic niche for successful implantation of disseminated cells $(10,11)$. These processes can occur early in the disease, an observation that has clinical (12) and biological support (13). Treatment of the primary tumor may not only have a positive effect on the localized consequences of tumor growth but also in the distant tumors that grow as metastasis (14). Considering this clinical and biological background, we proposed that cytoreduction of the primary tumor reduces or slows the progression of metastatic disease in a mouse model of mPCa. To address this question we previously generated a murine model of $\mathrm{CaP}$ that is surgically resectable without significant side-effects (15). This model consistently generates metastasis in a timedependent fashion and enables us to perform CS and study the behavior of tumors after treatment.

\section{Materials and methods}

Cells.PC3 cells were used for orthotopic injection. This cell line is derived from a bone metastasis of a human PCa patient and has a high tumorigenic and metastatic behavior when injected intravenously and orthotopically. These cells are androgenindependent and do not express PSA. In order to visualize the 
cells once injected in the mouse prostate, they were transduced with a gene that contains the sequence for firefly luciferase (GenTarget Inc. ${ }^{\circledR}$ ) (PC3-LUC cells) using viral particles pGreenFire1-LUC-CMV-EF1-Puro (cat. TR011VA-P; System Biosciences, Mountain View, CA, USA) diluted in Dulbecco's modified Eagle's medium (DMEM)/F12 10\% FBS with Polybrene (cat. H9268; Sigma-Aldrich) at a concentration of $5 \mu \mathrm{g} / \mathrm{ml}$. Cells were left overnight for infection, and puromycin was added $(1 \mu \mathrm{g} / \mathrm{ml})$ to select cells that were stably transduced. Integration was verified using qPCR and fluorescence.

Animals and orthotopic injection. NOD-SCID $\gamma$ mice (NOD. $\mathrm{Cg}-\mathrm{Prkd}^{\text {scid }} I l 2 \mathrm{rg}^{\text {tmlWjl}} / \mathrm{SzJ}$; Jackson Laboratory ${ }^{\circledR}$, Sacramento, CA, USA) were obtained from our High Safety Animal Facility (Faculty of Medicine, University of Chile) and maintained in a laminar flow room under specific pathogen-free conditions. All food, water and litter were sterilized prior to use. Temperature $\left(20-21^{\circ} \mathrm{C}\right)$ and humidity (50-60\%) were controlled. Daily light cycles were 12-h light and 12-h dark. Cages were changed fully once or twice a week. Animals were manipulated under sterile conditions. Orthotopic injection was performed using the anterior lobe of the mouse prostate as previously described (primer paper). All experiments with animals were approved by the Bioethics Committee of the Faculty of Medicine, University of Chile (protocol CBA\#0487 FMUCH).

Bioluminescence. Primary and metastatic tumor growth was followed using the IVIS Lumina II $^{\circledR}$ (Caliper Life Sciences, Hopkinton, MD, USA) system. Animals were anesthetized with a combination of ketamine and xylazine and injected intraperitoneally with $150 \mathrm{mg} / \mathrm{kg}$ of D-luciferin (potassium salt). Sixteen minutes after injection of D-luciferin, images were captured with the IVIS system. Images were taken in 5-7 day intervals until the end of the experiment.

Study design. Two groups of mice were used with 5 mice in each group. The first group (control) consisted of animals injected orthotopically in the prostate and then followed by luminescence imaging at regular intervals until completion of the experiments. These animals received no further manipulation. The second group (treatment) was injected orthotopically at time 0 and then subjected to $\mathrm{CS}$ of the primary tumor at day 30 in which metastatic growth had already occurred. These animals were followed by luminescence in the same manner as the first group until the end of the experiment.

$C S$. At day 30 post-injection of PC3-LUC cells, in the treatment group, animals were anesthetized and a midline incision was made on the skin and muscle, $1 \mathrm{~cm}$ caudal to the umbilicus. The prostate tumor and its associated seminal vesicle was identified, externalized and isolated. Using 6/0 absorbable suture, a ligature was made at the base of the anterior lobe, above the deferent duct. The prostate lobe was then cut over the ligature and removed with attention not to rupture the seminal vesicle. The same procedure was repeated for the left anterior prostatic lobe, along with its associated seminal vesicle. After checking for hemorrhages, the abdominal and skin incisions were closed in two planes. The tissue obtained from the CS was submerged in $10 \%$ neutral buffered formaldehyde. A luminescence image was taken after the procedure to ensure that no more than $5 \%$ of the tumor luminescence remained detectable. This percentage was considered a successful treatment.

Histology and immunohistochemistry. Tissues were fixed by immersion in neutral buffered formalin for $24 \mathrm{~h}$ and then they were trimmed and placed in histologic cassettes for dehydration, inclusion in paraffin and staining with hematoxylin and eosin $(\mathrm{H} \& \mathrm{E})$. Using the same tissues, an indirect immunoperoxidase method was performed as follows. Antigen retrieval was achieved by exposing the samples to $90^{\circ} \mathrm{C}$ for $30 \mathrm{~min}$ in citrate buffer $(10 \mathrm{mM}, \mathrm{pH} 6.0)$. Then the samples were incubated with the primary antibody [anti-human monoclonal anti-mitochondria (ab3298; Abcam, Cambridge, MA, USA), mouse monoclonal anti-CD-24 (CBL561; Chemicon, Temecula, CA, USA), rabbit monoclonal anti-CD-44 (ab51037; Abcam), mouse monoclonal anti-CD-133 (17A6.1; Millipore, Billerica, MA, USA) and mouse monoclonal anti-KI-67 (clone MIB-1; DakoCytomation, Glostrup, Denmark)] at $4^{\circ} \mathrm{C}$ overnight. The samples were treated with a streptavidin-biotin detection method (Histostain ${ }^{\circledR}$-Plus Bulk kit, Zymed ${ }^{\circledR}$, LAB-SA detection system and DAB-Plus Substrate kit; all from Invitrogen, Camarillo, CA, USA) followed by hematoxylin counterstaining. Images were obtained with a Leica ${ }^{\circledR}$ microscope, model DM3000. For semi-quantititive analysis of immunolabeling, a hybrid score (H-score) was calculated for each marker. This score resulted from determining the percentage of positive cells (from 0 to $100 \%$ ) and the intensity of staining (from 0-3 with 0, 1, 2, 3 grades corresponding to no staining, weak, medium and high intensity, respectively). These two numbers were then multiplied to obtain a score that ranged from 0 to 300. A proliferative index (PI) was calculated counting the number of KI-67 positive cells in three images obtained at $\mathrm{x} 400$ for each of three samples per group. The number of total and positive cells was obtained using the Image J software (ver. 1.48).

Statistics. Data were compiled and analyzed using Prism 6.0 software. Results were considered significantly different if $\mathrm{p}<0.05$, according to the specific statistical analysis used, as described in each figure legend.

\section{Results}

Primary tumor growth. After injection of PC3-Luc cells to both the control and treatment groups, we followed tumor growth by bioluminescence (Fig. 1A, Table I). The IVIS system has a level of background signal that is shown with a green line. This is the minimum signal that can be measured when an animal injected with cells but not with luciferin is photographed. CS was performed in the treatment group at day 30 (arrow). In all cases, the luminescence signal of the remnant tumors was $<5 \%$ of the original signal. On days 42 , $51,58,63$ and 70 , luminescence of the primary tumors in the treatment group was lower $(\mathrm{p}<0.01)$ than that of the control group demonstrating the effect of surgery on the tumor mass. The tumor mass was significantly reduced by the procedure but it was not eliminated, simulating the case of an advanced tumor which is not amenable to complete resection. At the end 
A

Days after injection of PC3-LUC cells

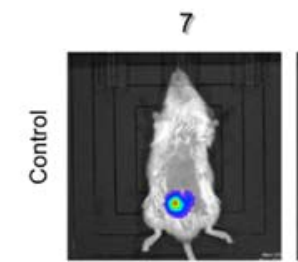

22

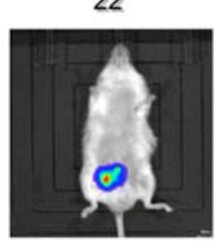

35
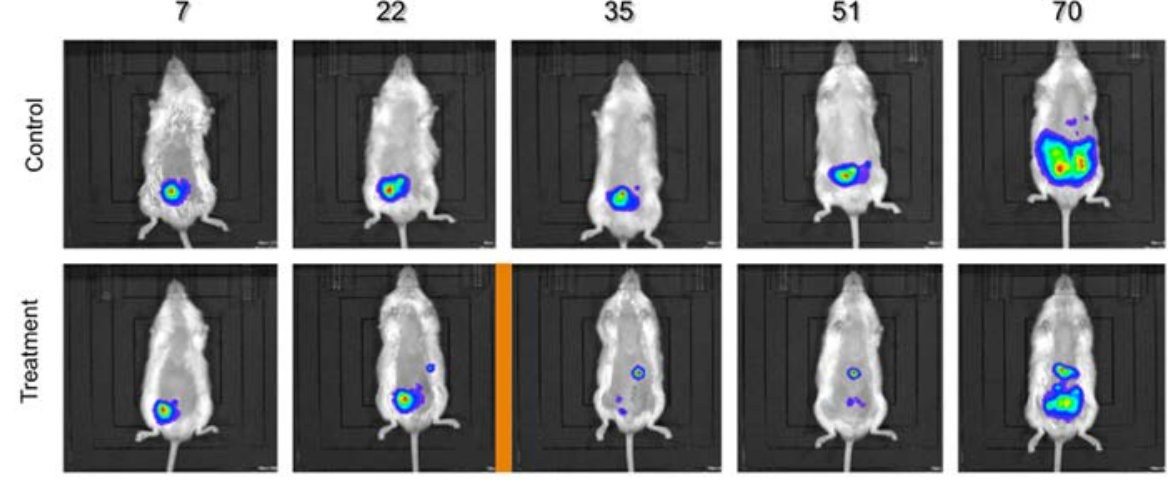

B

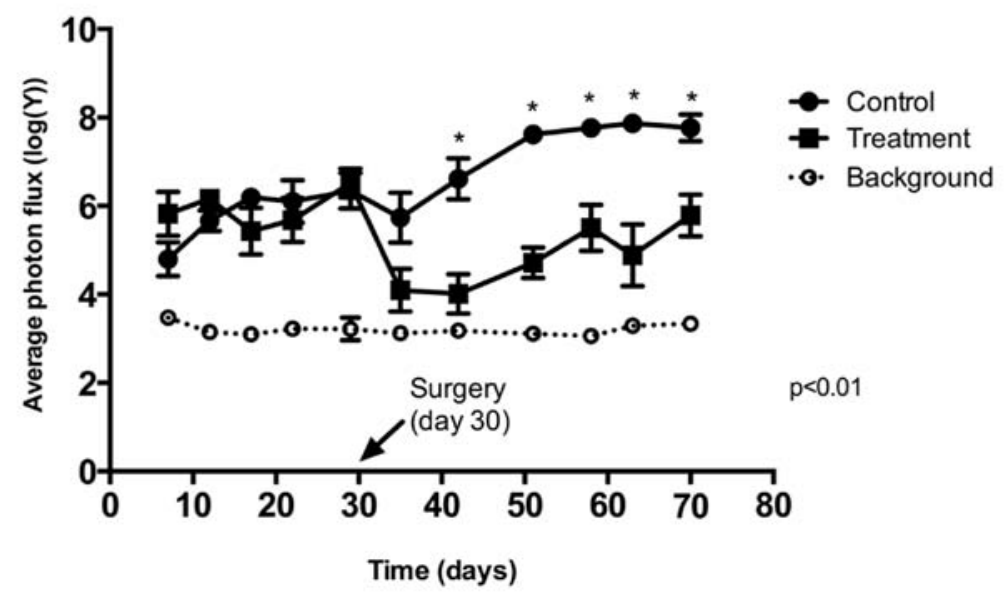

Figure 1. (A) Representative images of the mice in the control and treatment groups at 7, 22, 35, 51 and 70 days post-injection of PC3-LUC cells. Orange bar represents time of CS in mice from the treatment group. (B) Photon flux of the primary tumors in animals without treatment (control) and animals that were subjected to CS at day 30 post-injection of PC3-LUC cells (treatment). The dotted line shows the basal level of luminescence (background) which is the minimum signal obtained when measuring a mouse injected with PC3-LUC cells but without luciferin injection. Means were calculated based on a logaritmic transformation of the original data. Multiple Student's t-test; ${ }^{*} \mathrm{p}<0.01$, significant differences between control and treatment groups. Table I is a summary of these results.

Table I. Photon flux of the primary tumors.

$\frac{\text { Control }}{\text { Mean }} \frac{\text { Treatment }}{\text { Mean }}$

Day p-value $[\log ($ photon flux $)] \mathrm{SD}( \pm)[\log ($ photon flux $)] \mathrm{SD}( \pm)$

\begin{tabular}{rlllll}
\hline 7 & 0.140 & 4.798 & 0.851 & 5.821 & 1.108 \\
12 & 0.118 & 5.667 & 0.522 & 6.161 & 0.355 \\
17 & 0.217 & 6.193 & 0.494 & 5.429 & 1.173 \\
22 & 0.563 & 6.099 & 1.083 & 5.680 & 1.109 \\
29 & 0.743 & 6.346 & 0.899 & 6.522 & 0.729 \\
35 & 0.058 & 5.736 & 1.265 & 4.095 & 1.078 \\
42 & $0.004^{\mathrm{a}}$ & 6.612 & 1.036 & 4.012 & 1.001 \\
51 & $0.00003^{\mathrm{a}}$ & 7.619 & 0.169 & 4.715 & 0.766 \\
58 & $0.003^{\mathrm{a}}$ & 7.766 & 0.320 & 5.503 & 1.169 \\
63 & $0.003^{\mathrm{a}}$ & 7.866 & 0.408 & 4.884 & 1.560 \\
70 & $0.007^{\mathrm{a}}$ & 7.768 & 0.675 & 5.785 & 1.050 \\
\hline
\end{tabular}

Photon flux of the primary tumors [means and standard deviation (SD)] in animals without treatment (control) and animals that were subjected to cytoreductive surgery at day 30 post-injection of PC3-LUC cells (treatment). Means were calculated based on a logarithmic transformation of original data. Multiple Student's t-test; ${ }^{\mathrm{a}} \mathrm{p}<0.01$, significant differences. of the experiment (day 70) a rise in the level of luminescence was observed in the treatment group. This was still significantly lower than the level in the tumors in the control group and lower than the level in the tumors before surgery. In addition, in the treatment group, tumor size was less homogenous as demonstrated by their dispersion (SD, standard deviation; Table I).

Metastasis. Fig. 2 and Tables II and III show the general results for the metastases measured (Fig. 2A, Table II) and counted (Fig. 2B, Table III) by bioluminescence from day 42 after injection of the PC3-LUC cells until the end of the experiment at day 70. The size of the metastases (Fig. 2A), estimated by bioluminescence, was lower in the treatment group from day 51 until the end of the experiment. This size increased at the end of the experiment but failed to reach that of the control group. The effect on size was constant from day 42 until the end of the experiment showing that the main bulk of the primary tumor is needed to allow for metastasis to grow. The percentage of animals (Fig. 2B) that presented metastasis was similar in both cases, confirmed by contingency tables that showed no difference $(p>0.05)$ in the frequency of animals affected. This responds to the fact that 
Table II. Photon flux of metastatic tumors.

\begin{tabular}{|c|c|c|c|c|c|c|c|}
\hline \multirow[b]{2}{*}{ Day } & \multirow[b]{2}{*}{ p-value } & \multicolumn{3}{|c|}{ Control } & \multicolumn{3}{|c|}{ Treatment } \\
\hline & & $\begin{array}{c}\text { Mean } \\
{[\log (\text { photon flux })]}\end{array}$ & $\mathrm{SD}( \pm)$ & $\mathrm{N}$ & $\begin{array}{c}\text { Mean } \\
{[\log (\text { photon flux })]}\end{array}$ & $\mathrm{SD}( \pm)$ & $\mathrm{N}$ \\
\hline 42 & 0.135 & 5.894 & 1.252 & 3 & 4.803 & 1.015 & 3 \\
\hline 51 & $0.002^{\mathrm{a}}$ & 6.816 & 0.501 & 3 & 4.608 & 0.469 & 4 \\
\hline 58 & $0.018^{\mathrm{a}}$ & 6.232 & 0.946 & 5 & 4.770 & 0.462 & 4 \\
\hline 63 & $0.003^{\mathrm{a}}$ & 6.692 & 1.109 & 5 & 4.801 & 0.439 & 4 \\
\hline 70 & $0.005^{\mathrm{a}}$ & 7.120 & 0.960 & 5 & 5.441 & 0.966 & 5 \\
\hline
\end{tabular}

Photon flux of metastatic tumors in animals without treatment (control) and animals that were subjected to cytoreductive surgery at day 30 post-injection of PC3-LUC cells (treatment). Means were calculated based on a logarithmic transformation of original data. Multiple Student's t-test. ${ }^{a} \mathrm{p}<0.05$, significant differences between control and treatment groups.

Table III. Number of metastatic tumors.

\begin{tabular}{|c|c|c|c|c|c|c|c|}
\hline \multirow[b]{2}{*}{ Day } & \multirow[b]{2}{*}{$\mathrm{p}$-value } & \multicolumn{3}{|c|}{ Control } & \multicolumn{3}{|c|}{ Treatment } \\
\hline & & Mean & $\mathrm{SD}( \pm)$ & $\mathrm{N}(\%)$ & Mean & $\mathrm{SD}( \pm)$ & $\mathrm{N}(\%)$ \\
\hline 42 & 0.600 & 1.33 & 0.58 & $3(60)$ & 2.00 & 1.00 & $3(60)$ \\
\hline 51 & 0.726 & 2.67 & 2.08 & $3(60)$ & 2.25 & 1.50 & $4(80)$ \\
\hline 58 & 0.296 & 3.60 & 1.34 & $5(100)$ & 2.50 & 1.91 & $4(80)$ \\
\hline 63 & $0.003^{\mathrm{a}}$ & 5.80 & 1.30 & $5(100)$ & 2.50 & 1.91 & $4(80)$ \\
\hline 70 & $0.012^{\mathrm{a}}$ & 5.80 & 1.30 & $5(100)$ & 3.20 & 1.79 & $5(100)$ \\
\hline
\end{tabular}

Number of metastatic tumors in animals without treatment (control) and animals that were subjected to cytoreductive surgery at day 30 post-injection of PC3-LUC cells (treatment). Multiple Student's t-test. ${ }^{a} \mathrm{p}<0.05$, significant differences between control and treatment groups.

surgery was performed in animals that already had metastatic dissemination and therefore, surgery did not revert the process but only delayed it. Although the number of metastases was similar on days 42, 51 and 58, animals that received surgery (treatment group) had a lower number of metastases at the end of the experiment (days 63 and 70). This suggests that surgery of the primary tumor prevents the growth of new metastases. This result was also observed when counting metastases by microscopy (Fig. 3).

Necropsy and histology. The most frequent site affected by metastasis was the pancreas. It was followed by the hilum of the liver, spleen and stomach, the hilum of the kidney, mesentery, liver (parenchyma) and kidney (parenchyma). All mesenteric tumors were associated with a mesenteric vessel. None of the animals had tumors in the abdominal wall muscles or diaphragm, nor on the antimesenteric surface of the intestine, all of which were considered signs of carcinomatosis. Hence, all observed metastatic tumors were the result of circulatory dissemination. No apparent difference was noted in the histology of metastasis or the primary tumor when comparing both groups. This morphology was described in a previous study (15). Smaller tumors had a solid growth with scant amounts of stroma. Larger tumors had more stroma and

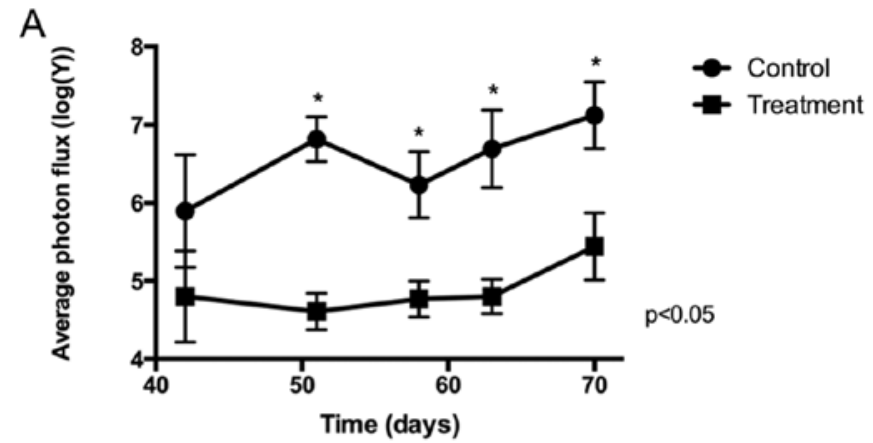

B

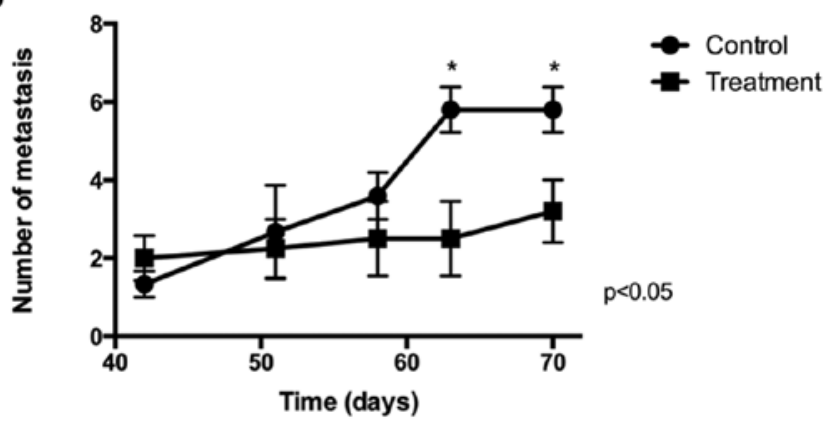

Figure 2. (A) Photon flux of metastatic tumors in animals without treatment (control) and animals that were subjected to cytoreductive surgery (CS) at day 30 post-injection of PC3-LUC cells (treatment). Means were calculated based on a logaritmic transformation of original data. (B) Number of metastatic tumors in animals without treatment (control) and animals that were subjected to CS at day 30 post-injection of PC3-LUC cells (treatment). Multiple Student's t-test. ${ }^{*} \mathrm{p}<0.05$, significant differences between control and treatment groups.

some had the formation of pseudoacinar structures. Treatment had no effect on the microscopic structure of the tumors.

CD-24, CD-44, CD-133 immunoreactivity and PI. Fig. 4 contains a summary of these results. A hybrid score (H-score) was used as a combined parameter of staining intensity and percentage of stained cells. In this analysis, tumors resected from animals at day 30 (treatment group) were included and represent the marker status of the primary tumors before surgery. CD-24 immunolabeling was high on all primary tumors, including the tumors resected on day 30 in the treat- 
A

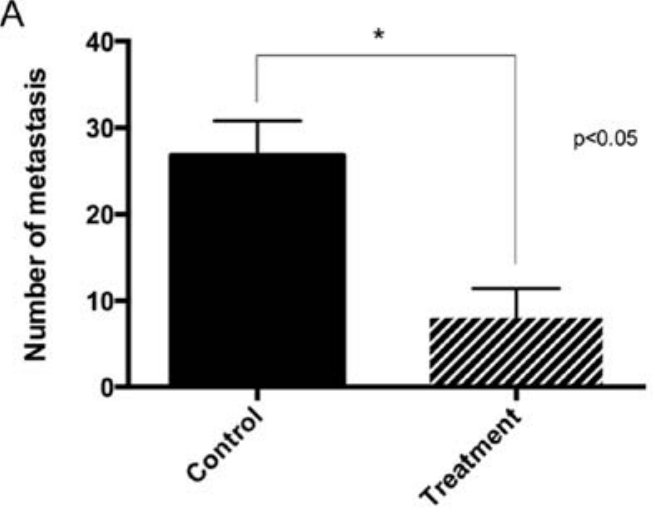

B

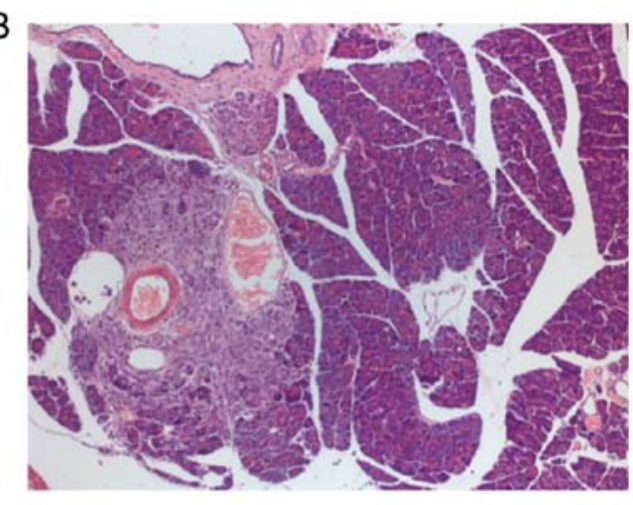

Figure 3. (A) Number of metastases counted by microscopy at the end of the experiment. Paired Student's t-test. p<0.05. (B) Representative image of a pancreatic metastasis.

ment group. In contrast, in both groups, CD-24 H-score was lower in the metastatic tumors. There was no difference between $\mathrm{H}$-score in metastasis from the control and treatment group. CD-44 immunolabeling was high in all tumors and no differences were observed between them. CD-133 immunolabeling was higher in all tumors of the treatment group compared to the tumors in the control group. Interestingly, CD-133 H-score in the resected tumors (treatment group) was similar to the one observed in tumors in the control group.

The PI (Fig. 5) was calculated as a percentage of KI-67 positive cells in $x 400$ fields. We compared the PI between primary and metastatic tumors in both groups. When comparing PI in the primary tumors, a higher percentage of KI-67-positive cells was found in the treatment group. These tumors arose from the remaining cells after CS suggesting that this is an effect of surgical treatment. Similarly, PI was higher in metastatic tumors of the treatment group versus metastases of the control group. These results suggest that proliferation was activated in the metastatic tumors from mice that received surgery. The effect of surgery on proliferation was, therefore, observed in all tumors confirming a systemic effect of treatment. When we compared PI between the primary tumors of the control group (day 70) with those resected at day 30 from mice in the treatment group (before any effect of surgery was possible) no difference was found, further indicating that a higher proliferative activity was a consequence of the surgical procedure.

\section{Discussion}

Surgical removal of the primary tumor as a treatment for metastatic cancer is an old idea that has been revisited throughout the history of oncology (16). Several studies in the 70's and 80's suggested that this treatment results in an increased number of metastases $(17,18)$. More recently, some evidence suggests that treatment of the primary tumor may activate angiogenesis, which in turn, may activate dormant metastatic cells (19). In the present study the observed effect of CS was a reduction in size and number of metastatic foci when compared with the non-treated mice. This effect suggests that surgery not only slows down the development of already established metastases but also reduces the number of new metastases, probably arising from dormant sites. Escaping dormancy depends on several factors, including angiogenesis, appropriate interaction with the local microenvironment, response to immune surveillance, and cancer stem cell presence $(20,21)$. There is ample evidence showing a promotive role of the primary tumor in preparing the microenvironment or pre-metastatic niche and facilitating survival and proliferation at distant sites $(10,11)$. This effect can be related to the mobilization of bone marrow-derived hematopoietic progenitor cells (11), the upregulation of extracellular matrix ligands and the expression of chemoattractants (22). All these factors may regulate the angiogenic-dependent dormancy status (23). Our results are in accordance with these observations. Another mechanism proposed for the primary tumor to distantly regulate the pre-metastatic niche is the delivery of microvesicles that can modify distant cells by transferring several classes of molecules, such as microRNAs (24). microRNAs have been proposed as regulators of several steps involved in the metastatic cascade, particularly regulating the occurrence of oligometastatic disease (25). In this context, the removal of the bulk mass of the primary tumor may alter delivery of these molecules, resulting in a less aggressive presentation. This hypothesis must be tested in an appropriate setting. Another important mechanism regarding tumor development is the role of cancer stem cells in initiating primary and metastatic tumors. To address this issue we evaluated expression of CD-24, CD-44 and CD-133 markers by immunolabeling. We found that the primary and metastatic tumors in the treated group had low CD-24 and high CD-44 and CD-133 expression compared to levels in the tumors from the non-treated animals. This combination is suggestive of a higher presence of less differentiated cells with high expression of stem cell markers. This denotes a possible switch in differentiation markers. Potential roles for CSCs are initiation of tumors, resistance to therapy and origination of metastasis $(26,27)$. The presence of CSC markers suggests that after surgical trauma more of these cells are able to induce the growth of the remaining tumor. This may be happening in metastasis as well, suggesting that after surgery, CSCs may have a role in repopulating the tumors (either metastatic or primary) rather than generating new metastatic foci. 


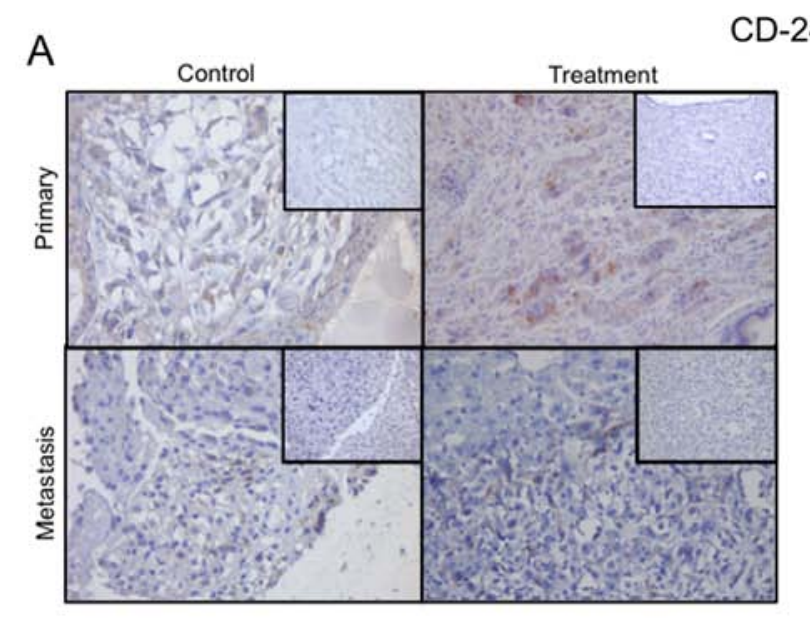

CD-24
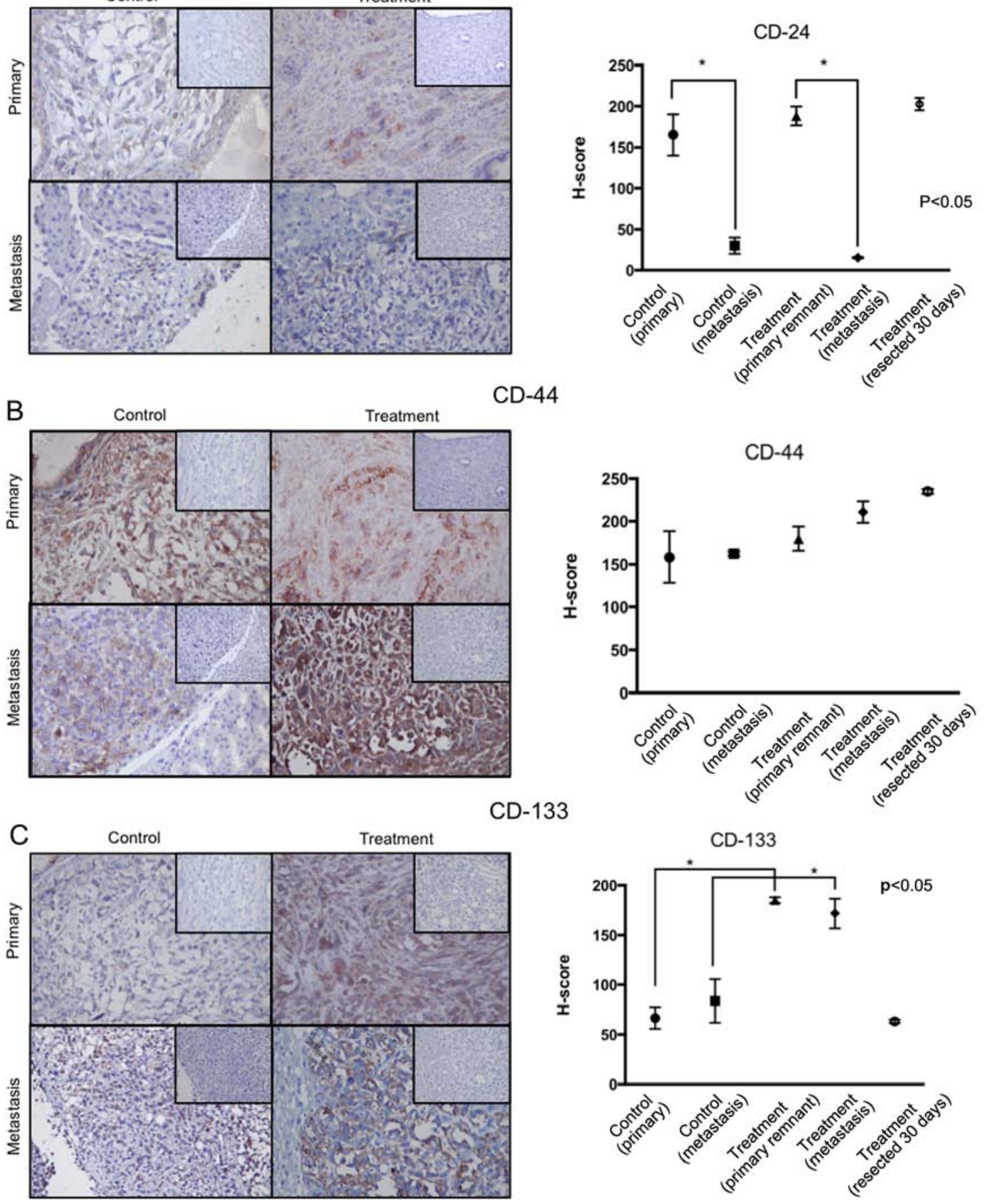

Figure 4. Representative images of (A) CD-24, (B) CD-44 and (C) CD-133 expression along with immunolabeling by determining H-score (original magnification, $\mathrm{x} 400$ ). In the images, boxes represent negative controls for each marker (original magnification, $\mathrm{x} 100$ ). Graphs show H-score in tumors from the control group (primary and metastatic, respectively) and from tumors in the treatment group (primary, metastatic and the resected tumors at surgery). Original magnification in all photomicrographs, $\mathrm{x} 400$. ANOVA and multiple Student's t-tests. ${ }^{*} \mathrm{p}<0.05$.

The role of immunity has been proposed to explain a potential negative effect of surgery. Some authors have suggested that surgery-related stress results in suppression of cellular mediated immunity, allowing metastatic development $(28,29)$. These authors propose that measures must be taken to avoid a perioperative immune depressive state, which may lead to uncontrolled metastatic development. However, they highlight that this effect probably occurs mainly in early stages of tumor development, before the immune system is co-opted by the tumor to develop tolerance (28). In this study we used NOD-SCID- $\gamma$ mice, which have severely dysfunctional innate and adaptive immunity (30) and hence have no immune barrier for tumor development. This is confirmed by the rapid development of primary and metastatic tumors in non-treated animals. However, in this experiment the effect of surgery on metastatic development was readily observable, suggesting that maximum metastatic growth in these mice depended not only on the lack of immune response but also on the presence 


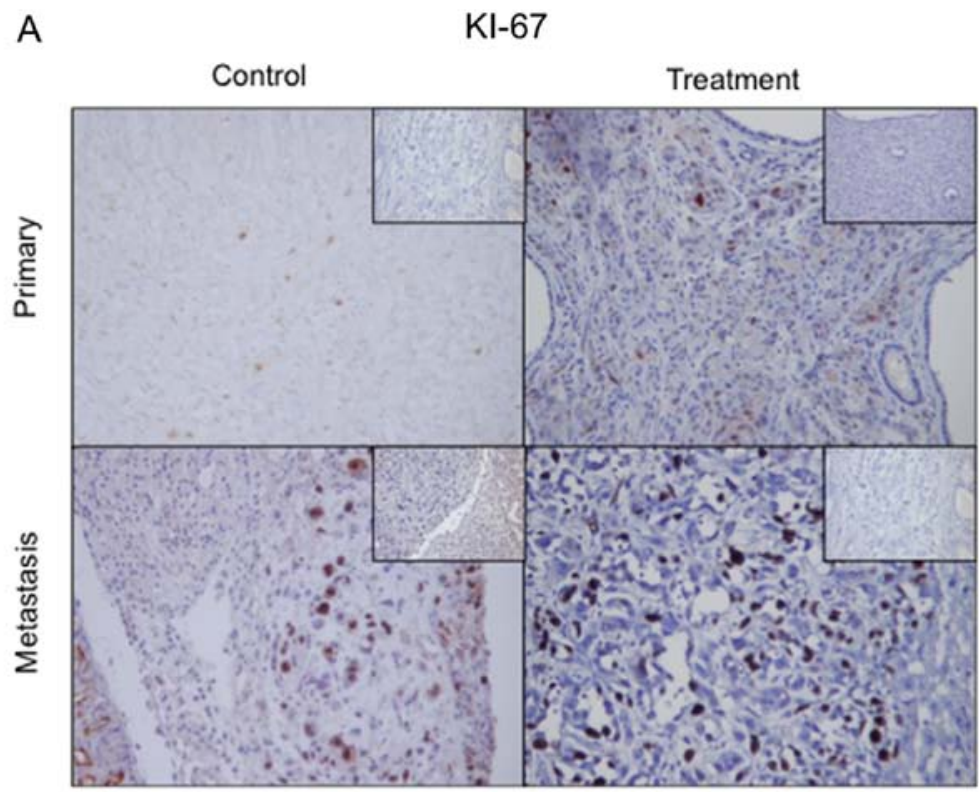

B

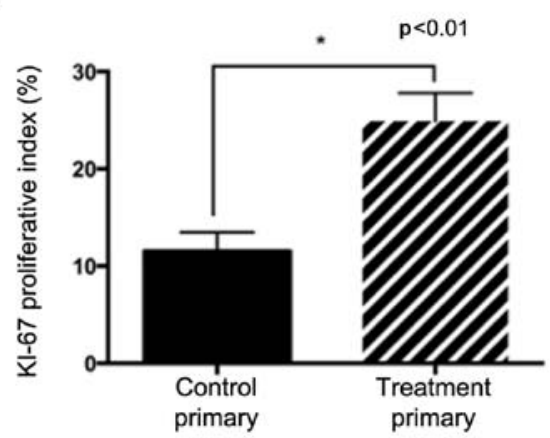

C

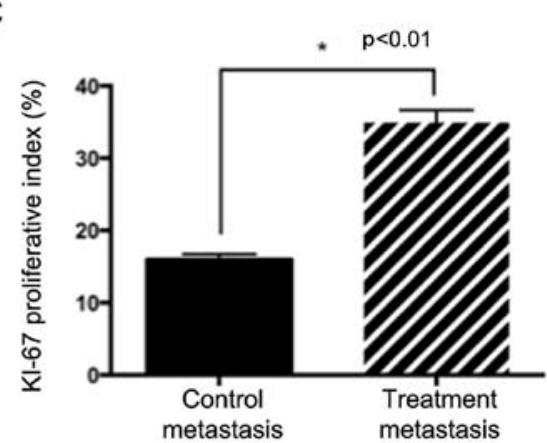

Figure 5. (A) Representative images of KI-67 labeling in primary and metastatic tumors from mice injected with PC3-LUC cells without treatment (control) and with cytoreductive surgery (CS) at day 30 (treatment). (B) The proliferative index (PI) (\%) in the same animals, comparing primary tumors between groups. (C) PI in the same animals comparing metastatic tumors. Unpaired t-test. "p $<0.01$.

of a fully developed primary tumor. Therefore, we conclude that the effect of surgery is, at least in part, independent of the immune status.

We chose to perform CS in contrast to complete removal of the tumor as this is more realistic in the context of a $\mathrm{PCa}$ T3 tumor and it is similar to other cancers, notably ovarian cancer (31). Our results show an important reduction in the luminescence signal in primary tumors as a consequence of treatment. After this reduction, a slow relapse occurred. The relapsed tumors in the treatment group did not reach the size of the primary tumors of the non-treated animals during the time of the experiment. Furthermore, they did not reach the size of the tumors before surgery. Although we saw a higher PI in the relapsed tumors, this was not sufficient to overcome the overall effect of surgery. A similar tendency was observed in the metastatic tumors, where a higher PI was observed in metastases from the treated animals. Apparently this is a late effect from surgery and may translate to future tumor growth. The significant reduction in primary and metastatic tumor size and numbers and the delay of their development, suggest that this treatment may translate to a survival benefit for $\mathrm{MPCa}$ patients, supporting the observations made in clinical trials (32).

CS is an accepted treatment for metastatic kidney (33), breast (34) and ovarian (31) cancer. This treatment depends on anatomical presentation and feasibility of surgery. This is clear in ovarian carcinoma in which patients with suboptimal tumor debulking derive no benefit from the surgical procedure and are only exposed to its complications (31). Furthermore, in PCa patients with locally advanced disease (T3 and/or N1), treatment of the primary tumor only achieves significant results when they are at high risk to die but not in those with a slow progression of the disease (35). If CS is to be used in $\mathrm{mPCa}$, pre-clinical and clinical evidence is necessary. There are several reports of retrospective studies that suggest this treatment may result in a survival benefit $(6,32)$. Recently, a large retrospective clinical study analyzed data from $\mathrm{PCa}$ patients in stage IV (M1) that had definitive treatment of the prostate tumor (5). The conclusion of this study suggests that there is a survival benefit for this treatment option. However, an editorial comment about this article points out that this treatment modality is not supported by any published guidelines and that more evidence is necessary to recommend the treatment of the primary tumor in mPCa patients outside of a clinical trial (4); moreover, concludes that well-thought-out clinical trials need proper patient selection (4). In agreement with this, here we showed in a pre-clinical model, that CS of the primary tumor alone can reduce metastatic burden and may translate to a survival benefit for cancer patients. Current 
modalities for treatment of mPCa patients include mainly androgen blockage and, when resistance develops, variable application of chemotherapy and radiotherapy (3). Surgery of the primary tumor may be a useful addition to these sequential approaches with the main goal of prolonging survival. Here we show evidence that may promote the design of prospective studies that address this subject.

\section{Acknowledgements}

The present study was supported by grants FONDECYT 1140417 (EAC) and 1151214 (HRC). F.F.C. and R.V. are granted with PhD CONICYT fellowships nos. 211006631 and 21100651, respectively. We thank Ms. Graciela Caroca and Mr. Miguel Sepulveda for their excellent technical assistance and Mr. Dagoberto Donoso for his expert animal handling.

\section{References}

1. Torre LA, Bray F, Siegel RL, Ferlay J, Lortet-Tieulent J and Jemal A: Global cancer statistics, 2012. CA Cancer J Clin 65: 87-108, 2015

2. Tangen CM, Hussain MH, Higano CS, Eisenberger MA, Small EJ, Wilding G, Donnelly BJ, Schelhammer PF, Crawford ED, Vogelzang NJ, et al: Improved overall survival trends of men with newly diagnosed M1 prostate cancer: A SWOG phase III trial experience (S8494, S8894 and S9346). J Urol 188: 1164-1169, 2012.

3. Valenca LB, Sweeney CJ and Pomerantz MM: Sequencing current therapies in the treatment of metastatic prostate cancer. Cancer Treat Rev 41: 332-340, 2015.

4. Chapin BF, McGuire SE and Aparicio A: Is treatment of the primary tumor in metastatic prostate cancer justified? Eur Urol 65: 1067-1068, 2014

5. Culp SH, Schellhammer PF and Williams MB: Might men diagnosed with metastatic prostate cancer benefit from definitive treatment of the primary tumor? A SEER-based study. Eur Urol 65: 1058-1066, 2014

6. Swanson G, Thompson I, Basler J and Crawford ED: Metastatic prostate cancer - Does treatment of the primary tumor matter? J Urol 176: 1292-1298, 2006.

7. Qin XJ, Ma CG, Ye DW, Yao XD, Zhang SL, Dai B, Zhang HL, Shen YJ, Zhu Y, Zhu YP, et al: Tumor cytoreduction results in better response to androgen ablation - a preliminary report of palliative transurethral resection of the prostate in metastatic hormone sensitive prostate cancer. Urol Oncol 30: 145-149, 2012.

8. Nguyen DX: Tracing the origins of metastasis. J Pathol 223: 195-204, 2011

9. Miyamoto DT, Sequist LV and Lee RJ: Circulating tumour cells - monitoring treatment response in prostate cancer. Nat Rev Clin Oncol 11: 401-412, 2014.

10. Sceneay J, Smyth MJ and Möller A: The pre-metastatic niche: Finding common ground. Cancer Metastasis Rev 32: 449-464, 2013.

11. Kaplan RN, Riba RD, Zacharoulis S, Bramley AH, Vincent L, Costa C, MacDonald DD, Jin DK, Shido K, Kerns SA, et al: VEGFR1-positive haematopoietic bone marrow progenitors initiate the pre-metastatic niche. Nature 438: 820-827, 2005.

12. Yu C, Shiozawa Y, Taichman RS, McCauley LK, Pienta K and Keller E: Prostate cancer and parasitism of the bone hematopoietic stem cell niche. Crit Rev Eukaryot Gene Expr 22: $131-148,2012$

13. Hüsemann Y, Geigl JB, Schubert F, Musiani P, Meyer M, Burghart E, Forni G, Eils R, Fehm T, Riethmüller G, et al: Systemic spread is an early step in breast cancer. Cancer Cell 13: $58-68,2008$.
14. Morgan SC and Parker CC: Local treatment of metastatic cancer - killing the seed or disturbing the soil? Nat Rev Clin Oncol 8: 504-506, 2011

15. Cifuentes FF, Valenzuela RH, Contreras HR and Castellón EA: Development of an orthotopic model of human metastatic prostate cancer in the NOD-SCID $\gamma$ mouse (Mus musculus) anterior prostate. Oncol Lett 10: 2142-2148, 2015.

16. Demicheli R, Retsky MW, Hrushesky WJ, Baum M and Gukas ID: The effects of surgery on tumor growth: A century of investigations. Ann Oncol 19: 1821-1828, 2008.

17. Clamage DM, Sanford CS, Vander AJ and Mouw DR: Effects of psychosocial stimuli on plasma renin activity in rats. Am J Physiol 231: 1290-1294, 1976.

18. Fisher B, Gunduz N, Coyle J, Rudock C and Saffer E: Presence of a growth-stimulating factor in serum following primary tumor removal in mice. Cancer Res 49: 1996-2001, 1989.

19. Camphausen K, Moses MA, Beecken WD, Khan MK, Folkman J and O'Reilly MS: Radiation therapy to a primary tumor accelerates metastatic growth in mice. Cancer Res 61: 2207-2211, 2001.

20. Aguirre-Ghiso JA: Models, mechanisms and clinical evidence for cancer dormancy. Nat Rev Cancer 7: 834-846, 2007.

21. Giancotti FG: Mechanisms governing metastatic dormancy and reactivation. Cell 155: 750-764, 2013.

22. Peinado H, Lavotshkin S and Lyden D: The secreted factors responsible for pre-metastatic niche formation: Old sayings and new thoughts. Semin Cancer Biol 21: 139-146, 2011.

23. Ghajar CM, Peinado H, Mori H, Matei IR, Evason KJ, Brazier H, Almeida D, Koller A, Hajjar KA, Stainier DY, et al: The perivascular niche regulates breast tumour dormancy. Nat Cell Biol 15: 807-817, 2013.

24. Antonyak MA and Cerione RA: Microvesicles as mediators of intercellular communication in cancer. Methods Mol Biol 1165: 147-173, 2014.

25. Uppal A, Ferguson MK, Posner MC, Hellman S, Khodarev NN and Weichselbaum RR: Towards a molecular basis of oligometastatic disease: Potential role of micro-RNAs. Clin Exp Metastasis 31: 735-748, 2014

26. Tu SM and Lin SH: Prostate cancer stem cells. Clin Genitourin Cancer 10: 69-76, 2012.

27. Shackleton M, Quintana E, Fearon ER and Morrison SJ: Heterogeneity in cancer: Cancer stem cells versus clonal evolution. Cell 138: 822-829, 2009.

28. Ben-Eliyahu S: The promotion of tumor metastasis by surgery and stress: Immunological basis and implications for psychoneuroimmunology. Brain Behav Immun 17 (Suppl 1): S27-S36, 2003.

29. Neeman E and Ben-Eliyahu S: Surgery and stress promote cancer metastasis: New outlooks on perioperative mediating mechanisms and immune involvement. Brain Behav Immun 30 (Suppl): S32-S40, 2013.

30. Shultz LD, Goodwin N, Ishikawa F, Hosur V, Lyons BL and Greiner DL: Human cancer growth and therapy in immunodeficient mouse models. Cold Spring Harb Protoc 2014: 694-708, 2014.

31. Narasimhulu DM, Khoury-Collado F and Chi DS: Radical surgery in ovarian cancer. Curr Oncol Rep 17: 16, 2015.

32. Faiena I, Singer EA, Pumill C and Kim IY: Cytoreductive prostatectomy: Evidence in support of a new surgical paradigm (Review). Int J Oncol 45: 2193-2198, 2014.

33. Krabbe LM, Haddad AQ, Westerman ME and Margulis V: Surgical management of metastatic renal cell carcinoma in the era of targeted therapies. World J Urol 32: 615-622, 2014.

34. Neuman HB, Morrogh M, Gonen M, Van Zee KJ, Morrow M and King TA: Stage IV breast cancer in the era of targeted therapy: Does surgery of the primary tumor matter? Cancer 116: 1226-1233, 2010.

35. Verhagen PC, Schröder FH, Collette L and Bangma CH: Does local treatment of the prostate in advanced and/or lymph node metastatic disease improve efficacy of androgen-deprivation therapy? A systematic review. Eur Urol 58: 261-269, 2010. 